Bio - grafia. Escritos sobre la Biología y su Enseñanza. ISSN 2027

Edición Extraordinaria. p.p. 1389-1399

Memorias del VIII Encuentro Nacional de Experiencias en Enseñanza de la Biología y la Educación Ambiental. III Congreso Nacional de Investigación en Enseñanza de la Biología.

\title{
EL JUEGO COMO HERRAMIENTA PARA LA ENSEÑANZA DEL FUNCIONAMIENTO DEL SISTEMA NERVIOSO EN LOS SERES VIVOS Y APORTE A LA SOLUCIÓN DE PROBLEMAS DE CONVIVENCIA EN EL AULA.
}

\section{GAME AS A TOOL FOR TEACHING THE PHYSIOLOGY OF THE NERVOUS SYSTEM IN LIVING AND CONTRIBUTION TO THE SOLUTION OF PROBLEMS OF COEXISTENCE IN THE CLASSROOM.}

\author{
Orozco Marín Yonier Alexander' ${ }^{1}$, Perdomo Gómez John Hanner²
}

\section{RESUMEN}

Tradicionalmente el entendimiento del juego en la educación se ha limitado al tiempo libre y las actividades de entretenimiento, limitando los aportes que el juego puede realizar a la construcción de ciudadanía y cultura en la sociedad y en el favorecimiento de construcción de aprendizajes en el área de las ciencias naturales por parte de los estudiantes. Esta investigación presentó como objetivo vincular el juego a las prácticas de enseñanza de la biología, específicamente del sistema nervioso con el fin de promover en los estudiantes aprendizajes sobre este concepto y aportar a la reflexión y solución entorno a problemas de convivencia escolar al interior de un curso de bachillerato de un colegio privado del municipio de Soacha Cundinamarca. Para ello se diseñó e implementó una actividad fundamentada didácticamente con el juego como herramienta, parte de un proceso de investigación escolar que el grupo se encontraba desarrollando. Como principales resultados se encontró que a través del juego los estudiantes revisan sus experiencias y relaciones con sus compañeros, identifican problemas en éstas y proponen alternativas y estrategias para superarlas hacia el cumplimiento de un objetivo de interés para el grupo, a la vez que, avanzan en la comprensión del concepto al construir analogías entre sistemas sociales y sistemas biológicos, y construir explicaciones sobre fenómenos de lo vivo valiéndose de conocimientos previos y su participación en experiencias lúdicas.

PALABRAS CLAVE: Juego y aprendizaje, juego y construcción de ciudadanía, enseñanza y aprendizaje de las ciencias, sistema nervioso.

\section{ABSTRACT}

Traditionally, the understanding of the game in education has been limited to leisure and entertainment activities, limiting the contributions that the game can make to building 
Bio - grafia. Escritos sobre la Biología y su Enseñanza. ISSN 2027

Edición Extraordinaria. p.p. 1389-1399

Memorias del VIII Encuentro Nacional de Experiencias en Enseñanza de la Biología y la Educación Ambiental. III Congreso Nacional de Investigación en Enseñanza de la Biología.

citizenship and culture in society and favoring construction of learning in the area natural science by students. This research presented aimed at linking the game to the practices of teaching biology, specifically the nervous system in order to promote students learning about this concept and contribute to the reflection and solving problems around school life into a year of high school at a private school in the municipality of Soacha Cundinamarca. For it, was designed and implemented based didactic activity with the game as a tool, part of a process of scholarly research that the group was developing. The main results found that through the game students review their experiences and relationships with peers, identify problems in these and propose alternatives and strategies to overcome them towards meeting a target of interest for the group, at the same time, advance in understanding the concept to build analogies between social systems and biological systems, and build explanations of phenomena of the living taking advantage of prior knowledge and participation in recreational experiences.

KEY WORDS: Game and learning, game and citizenship building, teaching and learning of science, nervous system.

\section{INTRODUCCIÓN}

En el "modelo clásico de educación" el "arte", la "creatividad" así como el "juego"; han sido categorías tomadas como el tiempo de no aprender o de no estudiar, desconociendo las riquezas que poseen como herramientas de formación y transformación de ciudadanía, por lo cual, implementarlas dentro del aula de clase, es romper con los esquemas clásicos de educación, autores como Vygotsky (2003) señalan la importancia del arte y el juego por sus aportes al aprendizaje en la infancia.

Por otra parte, el juego en la escuela se ha tomado como "la excusa" entre temas para permitir que los estudiantes se relajen o dispersen su mente, es decir, el juego se asocia siempre necesariamente al entretenimiento, postura que desconoce la potenciación de saberes o construcción de ellos por medio del juego. El juego como lo plantea Huizinga (1938), "se antepone a la cultura" lo cual hace referencia a que el juego, aporta a la construcción cultural y de ciudadanos, es decir, a entendernos dentro de un grupo social el cual nos influye y sobre el cual podemos ejercer influencia. No hay ninguna escuela que te enseñe a jugar, porque el juego está implícito en el desarrollo cognitivo del ser humano, las ciencias biológicas hablan del desarrollo y evolución del cuerpo, planteando "el Homo- 
Bio - grafia. Escritos sobre la Biología y su Enseñanza. ISSN 2027

Edición Extraordinaria. p.p. 1389-1399

Memorias del VIII Encuentro Nacional de Experiencias en Enseñanza de la Biología y la Educación Ambiental. III Congreso Nacional de Investigación en Enseñanza de la Biología.

Sapiens" de acuerdo a la importancia del juego podríamos hablar del "Homo-Ludens", "el hombre que juega" asociado a que el juego es un regalo de la evolución y es parte de la vida. Incluso, muchos animales como los primates juegan todo el tiempo para desarrollar habilidades para la vida.

El juego desde siempre ha formado parte de las dinámicas internas de los estudiantes. Los niños y niñas han aprendido a relacionarse con su entorno, crear relaciones, dinámicas sociales, por medio del juego. Desde lo cual, orientar los procesos educativos a través del juego, es preparar a los niños y niñas para la vida; favoreciendo la construcción de habilidades y conocimientos.

Respecto a este aspecto Melo y Hernández (2014) señalan que el docente debe tener claro que en el juego se manifiestan también aspectos relacionados con la conducta y la personalidad de los estudiantes. En los juegos reglados se aprende a compartir, a trabajar en equipo, a recibir orientaciones y sugerencias de otros, a seguir indicaciones y a cumplir una ruta específica para alcanzar los objetivos. También el juego puede favorecer la autonomía, la toma de decisiones y ayudar a establecer vínculos más afectivos con los espacios donde se desarrollan, como la escuela.

El juego resulta todo un desafío al considerarlo herramienta para la formación del ser humano. En palabras de Álvarez (2003) "El mundo moderno nos invita a replantear muchas acciones, una de ellas es el actuar docente ante la necesidad de formar hombres con capacidad de solución de problemas, habilidades comunicativas en una aldea global". Se puede decir entonces, que el rol docente es definitivo para promover esas acciones, que de una parte faciliten su labor en la enseñanza de contenidos, pero que también contribuyan en la formación de individuos con las habilidades necesarias para desenvolverse en un grupo social, valorando la diferencia de sus integrantes.

Específicamente en el campo de la enseñanza de las ciencias naturales se puede mencionar que la inclusión del juego en los procesos de enseñanza y de aprendizaje y la reflexión didáctica sobre este proceso es relativamente reciente. Autores como Melo y Hernández (2014) mencionan que en el ámbito escolar existen varias posibilidades didácticas para promover el juego en las clases de ciencias naturales que pueden, con una planificación y la estructura de una estrategia didáctica, mejorar la producción de conocimiento científico 
Bio - grafia. Escritos sobre la Biología y su Enseñanza. ISSN 2027

Edición Extraordinaria. p.p. 1389-1399

Memorias del VIII Encuentro Nacional de Experiencias en Enseñanza de la Biología y la Educación Ambiental. III Congreso Nacional de Investigación en Enseñanza de la Biología.

escolar, potenciar y desarrollar habilidades cognitivas y fomentar aprendizajes significativos.

En algunas investigaciones basadas en experiencias de aula se concluye que los juegos educativos permiten que los estudiantes aprendan los contenidos científicos de otra forma menos convencional y tradicional, desarrollen habilidades, actitudes y valores frente a problemáticas que se presentan en su entorno, además permite que sus conocimientos puedan ser utilizados y aplicados en otro tipo de contextos, los juegos educativos como herramienta didáctica en el aula permiten la interacción entre los estudiantes, el intercambio de saberes, la búsqueda conjunta de soluciones a problemas específicos como los ambientales, la socialización, el desarrollo de habilidades propias de la ciencia, la posibilidad de generar vínculos afectivos entre los mismos estudiantes y además con el profesor (Carmona \& Díaz, 2013).

Por otra parte, también se debe reconocer en el juego una herramienta para el aporte a la solución de problemas del aula como un grupo social y sus conflictos. Mejía y Martínez (2006) señalan que los juegos cooperativos son un medio efectivo para reducir los niveles de agresión de los estudiantes de las escuelas, si se respeta cada uno de sus componentes (cooperación, participación, aceptación y no competencia) aunque la consecución de éste logro se convierte en un proceso de enseñanza aprendizaje que requiere de mucho tiempo y constancia. Desde esta perspectiva el juego debe estar orientado al proceso y no al resultado, lo importante es participar cooperando con lo/as otros/as; favoreciendo la integración de los participantes desde el conocimiento, la escucha, el entendimiento y aceptación de las reglas y dinámica del juego (GEUZ, Centro Universitario de transformación de conflictos).

El objetivo de esta investigación fue el de caracterizar los aportes de una actividad orientada desde el juego cooperativo como posibilitador de experiencias significativas en el aula, en el aprendizaje sobre el funcionamiento del sistema nervioso en los seres vivos y la resolución de problemas de convivencia al interior del aula, en un grupo de estudiantes del grado octavo de la educación secundaria de la Institución Educativa Liceo Nueva Vida.

\section{METODOLOGIA}


Bio - grafia. Escritos sobre la Biología y su Enseñanza. ISSN 2027

Edición Extraordinaria. p.p. 1389-1399

Memorias del VIII Encuentro Nacional de Experiencias en Enseñanza de la Biología y la Educación Ambiental. III Congreso Nacional de Investigación en Enseñanza de la Biología.

Esta experiencia didáctica se desarrolló con 25 estudiantes del grado octavo de la Institución Educativa Liceo Nueva Vida ubicada en el barrio Altos de Cazuca del municipio de Soacha Cundinamarca.

Como parte de las actividades desarrolladas en el área de biología y química estos estudiantes se encontraban desarrollando un proyecto de investigación escolar sobre los efectos del Tetrahidrocannabinol (principio activo de la marihuana) en el sistema nervioso humano, en el cual a partir de actividades de reconocimiento de su territorio identificaron a la marihuana como la droga de distribución ilegal más consumida por los jóvenes del sector. Durante el desarrollo de las metodologías construidas por los estudiantes en orientación del docente para dar continuidad al proceso de investigación escolar, se presentaron problemas de convivencia al interior del aula como el aislamiento de integrantes del curso y agresión verbal y física entre diferentes compañeros, que impedían el desarrollo exitoso del proceso de investigación escolar y de las actividades de enseñanza y de aprendizaje.

De este modo, a partir de un proceso reflexivo del docente, se diseñó e implementó una actividad de una duración de 90 minutos que permitiera a los estudiantes reflexionar sobre estos problemas de convivencia y las estrategias para solucionarlos a través del juego, a la vez que la actividad favoreciera en los estudiantes complementar su proceso de investigación escolar y sus aprendizajes sobre el concepto de la importancia del sistema nervioso para los seres vivos.

La descripción de los momentos de la actividad se encuentra en la figura 1. La sesión fue registrada en video y fotografías, elementos usados para el análisis, así como las impresiones de la actividad registradas como docente en el diario de campo y los trabajos de los estudiantes productos de ésta (se seleccionaron al azar los trabajos de cuatro estudiantes). El análisis de la información se realizó desde un paradigma de investigación interpretativo desde el cual según Arnal \& Latorre (1992) el interés se centra en el estudio de los significados de las acciones humanas y de la vida social, enfatizando en la comprensión e interpretación de la realidad educativa desde los significados de las personas implicadas en los contextos educativos.

Figura 1. Momentos de la actividad implementada. 
Bio - grafia. Escritos sobre la Biología y su Enseñanza. ISSN 2027

Edición Extraordinaria. p.p. 1389-1399

Memorias del VIII Encuentro Nacional de Experiencias en Enseñanza de la Biología y la Educación Ambiental. III Congreso Nacional de Investigación en Enseñanza de la Biología.

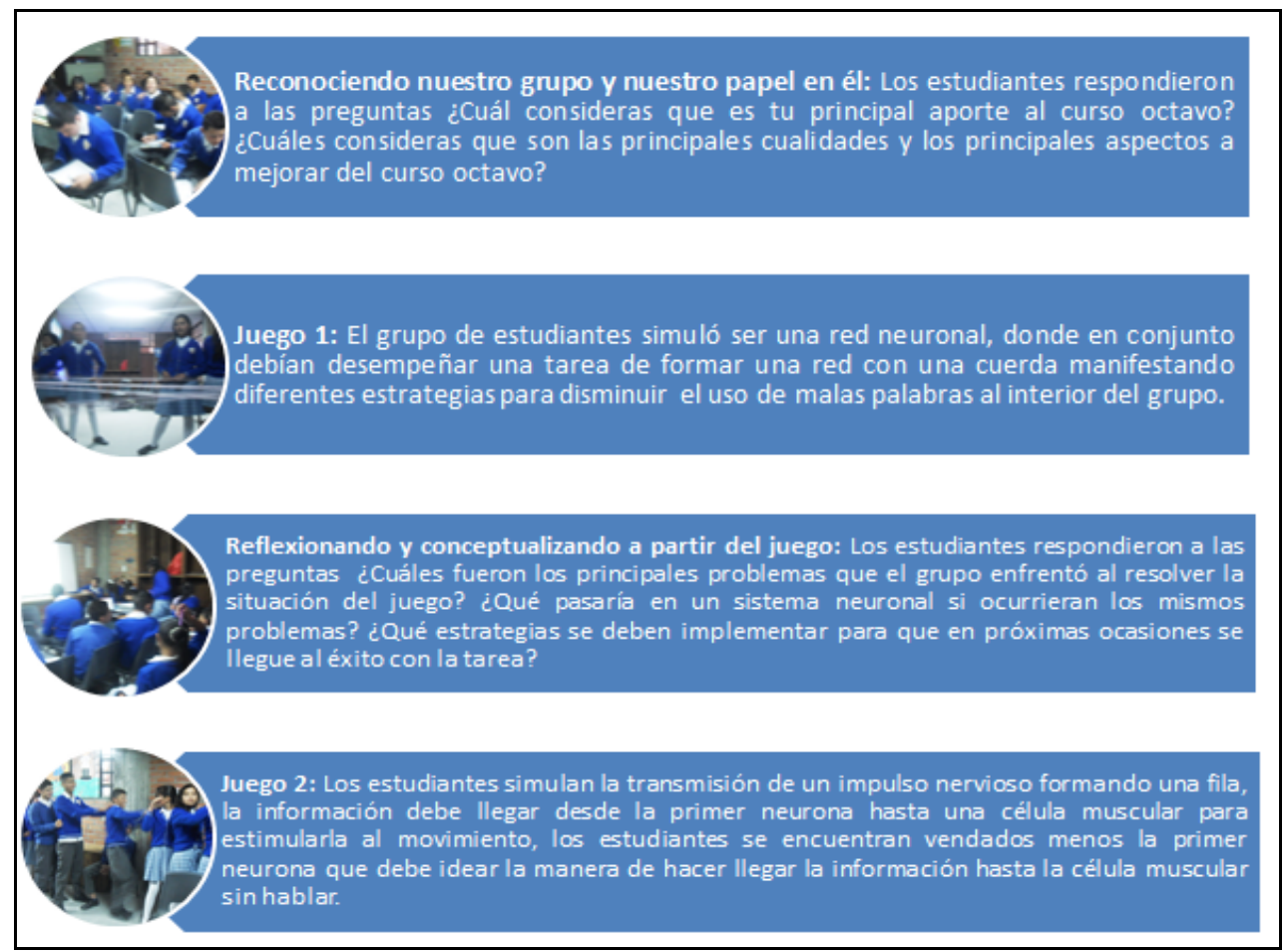

\section{RESULTADOS Y DISCUSIÓN}

\section{Reconociendo nuestro grupo y nuestro papel en él.}

Los estudiantes reconocen el grupo social al que pertenecen y con el cual aprenden, como un grupo "divertido para hacer las cosas, compañeristas se ayudan unos a otros y son cumplidos en algunas cosas (Est. 1)", un grupo en el cual hay "recocha, alegres, compañeristas y donde dejan copiar las tareas (Est. 2)", "activos (Est. 3)", un grupo con mucha "humildad (Est. 4)". Así mismo, como aspectos a mejorar del grupo mencionan "las groserías, la desobediencia, las malas influencias y los malos consejos (Est. 4)", "La gritería, el desorden y las peleas entre nosotros (Est. 1)", El uso de "apodos y las malas palabras entre los compañeros (Est. 3)".

Como integrantes de un grupo social, cumplimos un papel dentro de éste, no solo al dejarnos influenciar, sino también al ejercer influencia sobre este grupo, los estudiantes consideran que los aportes y papel dentro del grupo son "Yo aporto compañerismo, a los que necesitan amigos ahí siempre estoy (Est. 2)", "el compañerismo, la recocha, la indisciplina y el 
Bio - grafia. Escritos sobre la Biología y su Enseñanza. ISSN 2027

Edición Extraordinaria. p.p. 1389-1399

Memorias del VIII Encuentro Nacional de Experiencias en Enseñanza de la Biología y la Educación Ambiental. III Congreso Nacional de Investigación en Enseñanza de la Biología.

desorden (Est. 1)". En estas consideraciones de los estudiantes es notable como al referirse a su papel en el grupo destacan acciones en las cuales se relacionan con los demás, principalmente con aquellos que más comparten y con los que juegan espontáneamente, a pesar de que no se refieran necesariamente en los términos de juego, "yo aporto en mi grupo de amigos, los consejos, y planes positivos para levantar el ánimo (Est. 4)", de lo anterior es importante tener en cuenta que desde la niñez, el juego está presente de forma natural, pero con el paso del tiempo, las personas se alejan de lo que en un principio les divertía, para encontrar placer y diversión en otras cosas, es decir, que como a veces se cree, el juego no desaparece de las vidas de las personas, cambia, y adquiere otros sentidos.

\section{Jugando: Formando redes.}

Durante el desarrollo del juego 1, los estudiantes simularon cumplir cada uno el papel de una neurona, las cuales se integrarían en una red con el fin de cumplir una tarea. La tarea que el grupo decidió tratar en la actividad fue la del uso de groserías y malas palabras al interior del grupo, como un problema de convivencia que la mayoría consideraron.

Con el fin de estimular la comunicación entre los participantes del juego, al pasar la lana al siguiente compañero formando una red, se entendía que la lana correspondía al neurotransmisor que una neurona pre-sináptica enviaba a otra indicándole una tarea (una estrategia para resolver el problema del uso de las groserías), y esta neurona postsináptica debía recibir este mensaje (escuchar la estrategia) y ofrecer a otra neurona información nueva (un neurotransmisor), es decir, una estrategia nueva para ayudar a cumplir aquello que la anterior neurona le comunicó.

A sugerencia de los estudiantes la actividad contó con un tiempo para su realización, y a pesar de que el grupo contó con un tiempo antes del desarrollo de la actividad para organizar su estrategia, no hicieron uso de éste. Ya en el desarrollo de la actividad se hicieron presentes algunos problemas como gritos entre compañeros por falta de atención, falta de coordinación entre los participantes, un gran interés por parte de unos estudiantes y un desinterés por parte de otros en la actividad; lo cual ocasionó que el grupo no lograra cumplir el objetivo en el tiempo indicado, interpretando que la red neuronal no había conseguido desarrollar la tarea necesaria para el ser vivo del que hace parte. 
Bio - grafia. Escritos sobre la Biología y su Enseñanza. ISSN 2027

Edición Extraordinaria. p.p. 1389-1399

Memorias del VIII Encuentro Nacional de Experiencias en Enseñanza de la Biología y la Educación Ambiental. III Congreso Nacional de Investigación en Enseñanza de la Biología.

Este aspecto no se consideró como algo negativo, ya que se debe tener en cuenta, en el juego cooperativo lo importante no es el resultado, sino el proceso, y aquellos comportamientos manifestados durante el juego servirían posteriormente como objeto de reflexión por parte de los estudiantes sobre problemas de convivencia al interior del grupo y como ejemplos que abren la posibilidad para dar un abordaje didáctico al juego en la clase de ciencias (Melo \& Hernández, 2014) y conceptualizar los contenidos sobre el funcionamiento del sistema nervioso.

\section{Reflexionando y conceptualizando a partir del juego.}

Los estudiantes reflexionaron sobre los principales problemas que tuvieron al desarrollar la actividad, percibiendo estos como obstáculos para el desarrollo de tareas del grupo y el bienestar general de éste. Estos problemas corresponden a "la falta de comunicación y de atención (Est. 2)", "No nos concentrábamos en los demás compañeros y a veces no nos dábamos cuenta que la lana nos había llegado, entonces éramos muy lentos (Est. 1)", "los principales problemas que tuvimos fueron que algunos compañeros no pasaban la lana rápido - no escuchaban lo que decía el otro compañero y no sabían que responder (Est. 4)", "hubo mucho enredo y algunos no participaron en la actividad (Est. 3)". A partir de este ejercicio se puede mencionar contemplan debilidades en las habilidades socioemocionales del grupo (GEUZ, Centro Universitario de transformación de conflictos), refiriéndose estas a la capacidad de escuchar activamente y resolver conflictos constructivamente, aspectos claves para tener en cuenta en la planeación de juegos para el desarrollo de próximas actividades de enseñanza y de aprendizaje.

Al conceptualizar lo ocurrido en el juego con los problemas que esto traería para un organismo, los estudiantes hicieron uso de conocimientos construidos previamente en la secuencia didáctica y los organizaron para construir explicaciones sobre la importancia del sistema nervioso para un ser vivo y de su adecuado funcionamiento, explicaciones como las de la estudiante 3: "algunas neuronas no pasarían el neurotransmisor a otra neurona porque no entendieron el mensaje que le dejó la neurona anterior y si es muy importante la actividad el organismo se podría morir"; "Las neuronas, algunas se quemarían porque cuando una neurona no se usa y no recibe información se puede morir (Est. 1)", "Si las neuronas tuvieran esos problemas de comunicación, todo el sistema nervioso fallaría o sería muy lento, no habría sinapsis y esto sería muy difícil para el animal o el ser vivo hacer algo así (Est. 4)", finalmente el estudiante 2 menciona que "si un cuerpo funcionara como 
Bio - grafia. Escritos sobre la Biología y su Enseñanza. ISSN 2027

Edición Extraordinaria. p.p. 1389-1399

Memorias del VIII Encuentro Nacional de Experiencias en Enseñanza de la Biología y la Educación Ambiental. III Congreso Nacional de Investigación en Enseñanza de la Biología.

funcionamos en la actividad, las neuronas no cumplirían su actividad, ni su propósito, el cuerpo sería más lento y se podrían dañar las neuronas". Lo anterior, da muestra de que el juego también puede abrir la posibilidad de ofrecer situaciones que estimulen al estudiante a valerse de sus conocimientos para atribuir explicaciones e integrar conceptos que antes aparecían aislados en una explicación coherente y en la construcción de una analogía de un fenómenos biológico con una situación social.

Como posible mecanismos para dar solución a estos problemas al interior del grupo, los estudiantes proponen "hacer más actividades de agilidad, atención y puntería, para que fuéramos más agiles cuando tiremos la lana, una de atención para ver que dicen las demás neuronas (Est. 2)", es decir, los estudiantes ven en el juego una posibilidad para desarrollar habilidades que les permitirían una mejor comunicación en el grupo para cumplir mejor algunas tareas. Por otro lado, el estudiante 1 reconoce otras estrategias relacionadas con una mejor organización y planificación de las estrategias de forma grupal para tener éxito, "tomar el trabajo más enserio y organizarnos para escuchar todos atentamente lo que dice el otro, pensar una mejor estrategia grupal".

\section{Jugando: ¿Conseguimos mejorar?}

En el desarrollo del segundo juego los estudiantes se organizaron permitiendo que tres estudiantes asumieran el liderazgo en la coordinación del grupo, para lo cual establecieron compromisos individuales antes del desarrollo de la actividad y un código de comunicación para conseguir llevar el mismo mensaje desde la primera neurona hasta la célula muscular. Aunque al igual que en el anterior juego los estudiantes presentaron algunos problemas de comunicación y de descoordinación, es de destacar, que para esta ocasión estuvieron más cerca de conseguir el objetivo grupal del juego gracias a la organización inicial, los compromisos y códigos de comunicación establecidos. Lo cual, da muestra de la importancia de promover en próximas actividades una mayor participación del juego pensado y producto de la reflexión no solo de parte del profesor si no en conjunto con los estudiantes como estrategia didáctica para lograr vincularlo a contenidos como los efectos del THC en el sistema nervioso y el continuo trabajo sobre las formas de comunicación y organización dentro del grupo.

\section{CONCLUSIONES}


Bio - grafia. Escritos sobre la Biología y su Enseñanza. ISSN 2027

Edición Extraordinaria. p.p. 1389-1399

Memorias del VIII Encuentro Nacional de Experiencias en Enseñanza de la Biología y la Educación Ambiental. III Congreso Nacional de Investigación en Enseñanza de la Biología.

Las actividades pensadas desde el juego en los procesos de enseñanza y de aprendizaje de las ciencias pueden aportar a la reflexión por parte de los estudiantes en su rol como ciudadanos e integrantes de un grupo social del cual son influenciados y sobre el cual pueden ejercer influencia, promoviendo que estos revisen sus experiencias y relaciones con sus compañeros, identifiquen problemas en éstas y propongan alternativas y estrategias para superarlas hacia el cumplimiento de un objetivo de interés para el grupo, actividades que de ser diseñadas e implementadas con una mayor frecuencia y participación de los estudiantes no solo en su implementación, sino también en su planeación, pueden aportar significativamente a la solución de problemas de convivencia al interior del aula .

El juego puede constituirse en una herramienta que desde la enseñanza de las ciencias naturales, y que planeado y diseñado de una manera consiente y con un abordaje didáctico, participativo y crítico con los procesos de enseñanza y de aprendizaje y los contextos en los cuales los docentes y estudiantes se desenvuelven, aporte a la comprensión de conceptos de la biología (como la fisiología del sistema nervioso) al abrir las posibilidades para que los estudiantes construyan analogías entre sistemas sociales y sistemas biológicos, y construyan explicaciones sobre fenómenos sobre lo vivo valiéndose de conocimientos previos y su participación en experiencias lúdicas que se alejan de los esquemas tradicionales de enseñanza.

\section{BIBLIOGRAFÍA}

Álvarez, M. (2006) El juego en la Universidad: relato de una experiencia. Colombia 2006.

Carmona, V. \& Díaz, C. (2013) Una propuesta de material didáctico (juego de mesa) que favorece el proceso de enseñanza aprendizaje de la contaminación atmosférica y sus efectos en la salud humana. Trabajo de grado, Instituto de Educación y Pedagogía de la Universidad del Valle.

GEUZ, Centro Universitario de transformación de conflictos. Programas y estrategias para la convivencia escolar. España. Consultado en http://www.leioa.net/vive_doc/guia_convivencia_cast.pdf 
Bio - grafia. Escritos sobre la Biología y su Enseñanza. ISSN 2027

Edición Extraordinaria. p.p. 1389-1399

Memorias del VIII Encuentro Nacional de Experiencias en Enseñanza de la Biología y la Educación Ambiental. III Congreso Nacional de Investigación en Enseñanza de la Biología.

Hernández, R. \& Melo, M. (2014) El juego y sus posibilidades en la enseñanza de las ciencias naturales. Revista Innovación Educativa, v. 6, 66, p.p. 41-63.

Huizinga, J. (1938) Homo Ludens. Amsterdam University Press

Martínez, J. \& Mejía, E. (2006). El juego cooperativo, estrategia para reducir la agresión en los estudiantes escolares. Informe de práctica, Universidad de Antioquia, Colombia. Consultado

en http://viref.udea.edu.co/contenido/pdf/07_el_juego_cooperativo.pdf

Vygostsky, L. (2003) La imaginación y el arte en la infancia. Editorial Akal, edición 6, 128 páginas. 